\title{
The Dual QCD @ Work: 2018
}

\author{
Andrzej J. Buras* \\ ${ }^{1}$ TUM Institute for Advanced Study, Lichtenbergstr. 2a, D-85748 Garching, Germany
}

\begin{abstract}
The Dual QCD (DQCD) framework, based on the ideas of 't Hooft and Witten, and developed by Bill Bardeen, Jean-Marc Gérard and myself in the 1980s is not QCD, a theory of quarks and gluons, but a successful low energy approximation of it when applied to $K \rightarrow \pi \pi$ decays and $K^{0}-\bar{K}^{0}$ mixing. After years of silence, starting with 2014, this framework has been further developed in order to improve the SM prediction for the ratio $\varepsilon^{\prime} / \varepsilon$, the $\Delta I=1 / 2$ rule and $\hat{B}_{K}$. Most importantly, this year it has been used for the calculation of all $K \rightarrow \pi \pi$ hadronic matrix elements of BSM operators which opened the road for the general study of $\varepsilon^{\prime} / \varepsilon$ in the context of the SM effective theory (SMEFT). This talk summarizes briefly the past successes of this framework and discusses recent developments which lead to a master formula for $\varepsilon^{\prime} / \varepsilon$ valid in any extension of the SM. This formula should facilitate the search for new physics responsible for the $\varepsilon^{\prime} / \varepsilon$ anomaly hinted by 2015 results from lattice QCD and DQCD.
\end{abstract}

\section{Introduction}

Among the main stars of kaon flavour physics is the ratio $\varepsilon^{\prime} / \varepsilon$ that describes the amount of direct CP violation in $K_{L} \rightarrow \pi \pi$ decays relative to the indirect one. The latter one is related to $K^{0}-\bar{K}^{0}$ mixing, where also the $K_{L}-K_{S}$ mass difference, $\Delta M_{K}$, is an important quantity. Among rare kaon decays the most important roles these days are played by rare decays $K^{+} \rightarrow \pi^{+} v \bar{v}$ and $K_{L} \rightarrow \pi^{0} v \bar{v}$, soon to be measured by NA62 and KOTO experiments, respectively.

Various aspects of these decays and observables are reviewed at length in [1, 2]. Here we will concentrate our discussion on $\varepsilon^{\prime} / \varepsilon$ that has been measured already many years ago with the experimental world average from NA48 [3] and $\mathrm{KTeV}[4,5]$ collaborations given by

$$
\left(\varepsilon^{\prime} / \varepsilon\right)_{\exp }=(16.6 \pm 2.3) \times 10^{-4} .
$$

There are at least two reasons for concentrating on this ratio. First the situation of $\varepsilon^{\prime} / \varepsilon$ in the SM can be briefly summarized as follows:

- The analysis of $\varepsilon^{\prime} / \varepsilon$ by the RBC-UKQCD collaboration based on their lattice QCD calculation of $K \rightarrow \pi \pi$ matrix elements [6,7], as well as the analyses performed in [8,9] that are based on the same matrix elements but also include isospin breaking effects, find $\varepsilon^{\prime} / \varepsilon$ in the ballpark of $(1-2) \times 10^{-4}$. This is by one order of magnitude below the data, but with an error in the ballpark of $5 \times 10^{-4}$. Consequently, based on these analyses, one can talk about an $\varepsilon^{\prime} / \varepsilon$ anomaly of at most $3 \sigma$.

*e-mail: aburas@ph.tum.de 
- An independent analysis based on hadronic matrix elements from the Dual QCD (DQCD) approach $[10,11]$ gives a strong support to these values and moreover provides an upper bound on $\varepsilon^{\prime} / \varepsilon$ in the ballpark of $6 \times 10^{-4}$.

- A different view has been expressed in [12] where, using ideas from chiral perturbation theory, the authors find $\varepsilon^{\prime} / \varepsilon=(15 \pm 7) \times 10^{-4}$. While in agreement with the measurement, the large uncertainty, that expresses the difficulties in matching long distance and short distance contributions in this framework, does not allow for clear-cut conclusions. Consequently, values above $2 \times 10^{-3}$, that are rather unrealistic from the point of view of lattice QCD and DQCD, are not excluded in this approach.

Based on the results from RBC-UKQCD and the DQCD approach of 2015 a number of analyses have been performed in specific models beyond the SM (BSM) with the goal to obtain a sufficient upward shift in $\varepsilon^{\prime} / \varepsilon$ and thereby its experimental value. They are collected in Table 1 of [2]. An important limitation of these analyses is that they address the $\varepsilon^{\prime} / \varepsilon$ anomaly only in models in which new physics (NP) enters exclusively through modifications of the Wilson coefficients of SM operators. However, generally, BSM operators with different Dirac structures - like the ones resulting from tree-level scalar exchanges and leading to scalar and tensor operators - or chromo-magnetic dipole operators could play a significant role in $\varepsilon^{\prime} / \varepsilon$. Until recently, no quantitative judgment of the importance of such operators was possible because of the absence of even approximate calculations of the relevant hadronic matrix elements in QCD.

This brings me to the second reason for concentrating here on $\varepsilon^{\prime} / \varepsilon$. Namely, recently a significant theoretical progress has been made towards the general search for NP responsible for the hinted $\varepsilon^{\prime} / \varepsilon$ anomaly. This progress is based on the following analyses:

- the first to date calculations of the $K \rightarrow \pi$ and $K \rightarrow \pi \pi$ matrix elements of the chromomagnetic dipole operators by lattice QCD [13] and DQCD [14], respectively and in particular the calculation of $K \rightarrow \pi \pi$ matrix elements of all four-quark BSM operators, including scalar and tensor operators, by DQCD [15].

- derivation of a master formula for $\varepsilon^{\prime} / \varepsilon$ [16], which can be applied to any theory beyond the SM in which the Wilson coefficients of all contributing operators have been calculated at the electroweak scale. The relevant hadronic matrix elements of BSM operators used in this formula are from the DQCD, as lattice QCD did not calculate them yet, and the SM ones from lattice QCD.

- the first to date model-independent anatomy of the ratio $\varepsilon^{\prime} / \varepsilon$ in the context of the $\Delta S=1$ effective theory with operators invariant under QCD and QED and in the context of the Standard Model Effective Field Theory (SMEFT) with the operators invariant under the full SM gauge group [17].

The main goal of this talk is to review briefly these advances and to stress that they would not be possible in this decade without the existence of DQCD approach [10, 18-20].

We begin our presentation in Section 2 by recalling the general structure of the effective Hamiltonian valid in any BSM theory and summarizing the number of BSM operators one has to consider. In Section 3 we will briefly recall the main virtues of the DQCD approach. In this section we will stress the importance of the so-called meson evolution, an important ingredient of DQCD, which describes analytically very important dynamics at scales lower than $1 \mathrm{GeV}$. In Section 4 we will briefly review the present status of $\varepsilon^{\prime} / \varepsilon$ anomaly and in Section 5 we will present a master formula for $\varepsilon^{\prime} / \varepsilon$ that is valid in any theory beyond the SM [16]. In Section 6 we summarize the main lessons from the EFT and SMEFT analyses of $\varepsilon^{\prime} / \varepsilon$ in [17]. We conclude in Section 7 with an outlook for coming years. 


\section{Effective Hamiltonian for $K \rightarrow \pi \pi$ Beyond the SM}

The technology of operator product expansion and renormalization group can easily be extended to any model containing heavy particles and new interactions. Starting with a NP scale $\Lambda_{\mathrm{NP}}$ which could still be of order of a few TeV but also much larger, like $100 \mathrm{TeV}$, one can consecutively integrate out heavy particles and construct a series of effective theories until one reaches the electroweak scale. Below it only gluons, photon and SM quarks and leptons except for the top quark appear as dynamical degrees of freedom. Subsequently the known $\mathrm{RG}$ evolution down to scales relevant for a given decay is performed. The resulting effective Hamiltonian at these low energy scales has the following general structure:

$$
\mathcal{H}_{\mathrm{eff}}=\sum_{i} C_{i} O_{i}^{\mathrm{SM}}+\sum_{j} C_{j}^{\mathrm{NP}} O_{j}^{\mathrm{NP}} .
$$

The four objects appearing in this formula are as follows:

- $O_{i}^{\mathrm{SM}}$ are local operators present in the $\mathrm{SM}$ and $O_{j}^{\mathrm{NP}}$ are new operators having typically new Dirac structures, in particular scalar-scalar and tensor-tensor operators. They will play an important role soon.

- $C_{i}$ and $C_{j}^{\mathrm{NP}}$ are the Wilson coefficients of these operators. But whereas $C_{j}^{\mathrm{NP}}$ are only nonvanishing in the presence of NP,

$$
C_{i}=C_{i}^{\mathrm{SM}}+\Delta_{i}^{\mathrm{NP}}
$$

This means that in the presence of NP the Wilson coefficients of SM operators are generally modified.

The amplitudes for $K \rightarrow \pi \pi$ decays can now be written as follows

$$
\mathcal{A}(K \rightarrow \pi \pi)=\sum_{i} C_{i}(\mu)\left\langle\pi \pi\left|O_{i}^{\mathrm{SM}}(\mu)\right| K\right\rangle+\sum_{j} C_{j}^{\mathrm{NP}}(\mu)\left\langle\pi \pi\left|O_{j}^{\mathrm{NP}}(\mu)\right| K\right\rangle .
$$

The coefficients $C_{i}$ and $C_{j}^{\mathrm{NP}}$ can be calculated in the RG improved perturbation theory, although generally the analysis is rather complicated because of the presence of many operators that mix under renormalization. As far as QCD and QED corrections are concerned, they have been known already for 25 years at NLO [21-26] and for the BSM operators twoloop anomalous dimensions have been known [27, 28] for almost two decades. First steps towards NNLO predictions for $\varepsilon^{\prime} / \varepsilon$ have been made in [29-32] and further progress towards a complete NNLO result is under way [33].

In the context of the so-called SM effective theory not only QCD and QED effects below the electroweak scale but also electroweak effects above this scale and in particular the top quark Yukawa coupling have an important impact on the values of the coefficients $C_{i}$ and $C_{j}^{\mathrm{NP}}$ at the electroweak scale. But fortunately all these effects are already summarized for EFT in $[34,35]$ and for SMEFT in [36-38].

Until recently only the first sum in (4) could be evaluated because the matrix elements of the new operators were unknown. The new development is the calculation of the matrix elements of all BSM four-quark operators using DQCD in the SU(3) chiral limit [15]. As it will still take some time before corresponding results in lattice QCD will be available, already these approximate results from DQCD can teach us a lot about the relevance of various operators. Putting aside the chromomagnetic dipole operator, for a given chirality there are 13 BSM four-quark operators in the EFT and 7 BSM four-quark operators in the SMEFT for which hadronic matrix elements have to be calculated. This counting is demonstrated in [15] for the case of $(u, d, s)$ quarks. The full list of operators including those with $(c, b)$ quarks can be found in Table 1 of [16]. 


\section{Basics of Dual QCD Approach}

\subsection{Basic framework}

This analytic approach to $K \rightarrow \pi \pi$ decays and $K^{0}-\bar{K}^{0}$ mixing originated in the ideas of 't Hooft and Witten [39-42] based on large number $N$ of colours. In this limit QCD at very low momenta becomes a theory of weakly interacting mesons with the coupling $O(1 / N)$ and in particular in the strict large $N$ limit becomes a free theory of mesons simplifying the calculations significantly. With non-interacting mesons the factorization of matrix elements of four-quark operators into matrix elements of quark currents and quark densities used adhoc in the 1970s and early 1980s is automatic and can be considered as a property of QCD in this limit. But factorization cannot be the whole story as the most important QCD effects related to asymptotic freedom are related to non-factorizable contributions generated by exchanges of gluons. In DQCD this role is played by meson loops that represent dominant non-factorizable contributions at the very low energy scale. Calculating these loops one discovers then that factorization in question does not take place at values of $\mu \geq 1 \mathrm{GeV}$ at which Wilson coefficients are calculated but rather at very low momentum transfer between coloursinglet currents or densities.

The explicit calculation of the contributions of pseudoscalars to hadronic matrix elements of local operators is based on a truncated chiral Lagrangian describing the low energy interactions of the lightest mesons [43-45]

$$
L_{t r}=\frac{F^{2}}{8}\left[\operatorname{Tr}\left(D^{\mu} U D_{\mu} U^{\dagger}\right)+r \operatorname{Tr}\left(m U^{\dagger}+\text { h.c. }\right)-\frac{r}{\Lambda_{\chi}^{2}} \operatorname{Tr}\left(m D^{2} U^{\dagger}+\text { h.c. }\right)\right]
$$

where

$$
U=\exp \left(i \sqrt{2} \frac{\Pi}{F}\right), \quad \Pi=\sum_{\alpha=1}^{8} \lambda_{\alpha} \pi^{\alpha}
$$

is the unitary chiral matrix describing the octet of light pseudoscalars. The parameter $F$ is related to the weak decay constants $F_{\pi} \approx 130 \mathrm{MeV}$ and $F_{K} \approx 156 \mathrm{MeV}$ through

$$
F_{\pi}=F\left(1+\frac{m_{\pi}^{2}}{\Lambda_{\chi}^{2}}\right), \quad F_{K}=F\left(1+\frac{m_{K}^{2}}{\Lambda_{\chi}^{2}}\right),
$$

so that $\Lambda_{\chi} \approx 1.1 \mathrm{GeV}$. The diagonal mass matrix $m$ involving $m_{u}, m_{d}$ and $m_{s}$ is such that

$$
r(\mu)=\frac{2 m_{K}^{2}}{m_{s}(\mu)+m_{d}(\mu)},
$$

with $r(1 \mathrm{GeV}) \approx 3.75 \mathrm{GeV}$ for $\left(m_{s}+m_{d}\right)(1 \mathrm{GeV}) \approx 132 \mathrm{MeV}$.

In order to calculate the matrix elements of the local operators in question we need meson representations of colour-singlet quark currents and densities. They are directly obtained from the effective Lagrangian in (5) and are given respectively as follows

$$
\begin{gathered}
\bar{q}_{L}^{b} \gamma_{\mu} q_{L}^{a}=i \frac{F^{2}}{8}\left\{\left(\partial_{\mu} U\right) U^{\dagger}-U\left(\partial_{\mu} U^{\dagger}\right)+\frac{r}{\Lambda_{\chi}^{2}}\left[\left(\partial_{\mu} U\right) m^{\dagger}-m\left(\partial_{\mu} U^{\dagger}\right)\right]\right\}^{a b}, \\
\bar{q}_{R}^{b} q_{L}^{a}=-\frac{F^{2}}{8} r\left[U-\frac{1}{\Lambda_{\chi}^{2}} \partial^{2} U\right]^{a b},
\end{gathered}
$$

with $U$ turned into $U^{\dagger}$ under parity. 
At the tree level, corresponding to leading order in $1 / N$, one uses these representations to simply express the operators in terms of the meson fields and expands the matrix $U$ in powers of $1 / F$. For $K \rightarrow \pi \pi$ decays the relevant contribution to hadronic matrix elements is read off from terms involving only one kaon and two pions. However, in the large $N$ limit the hadronic matrix elements factorize and in order to combine them with the Wilson coefficients loops in the meson theory have to be calculated. In contrast to chiral perturbation theory, in DQCD a physical cut-off $\Lambda$ is used in the integration over loop momenta. As discussed in detail in $[18,20]$ this allows to achieve much better matching with short distance contributions than it is possible in chiral perturbation theory which uses dimensional regularization. The cutoff $\Lambda$ is typically chosen around $0.7 \mathrm{GeV}$ when only pseudoscalar mesons are exchanged in the loops [18] and can be increased to $0.9 \mathrm{GeV}$ when contributions from lowest lying vector mesons are taken into account as done in [20]. These calculations are done in a momentum scheme but as demonstrated in [20] they can be matched to the commonly used NDR scheme. Once this is done it is justified to set $\Lambda \approx \mu$. We ask sceptical readers to study a detailed exposition of DQCD in [20] where also the differences from the usual chiral perturbation calculations are emphasized.

\subsection{Grand view of DQCD}

The application of DQCD to weak decays consists in any model of the following steps:

Step 1: At $\Lambda_{\mathrm{NP}}$ one integrates out the heavy degrees of freedom and performs the RG evolution including Yukawa couplings and all gauge interactions present in the SM down to the electroweak scale. This evolution involves in addition to SM operators also BSM operators. This is SMEFT.

Step 2: At the electroweak scale $W, Z$, top quark and the Higgs are integrated out and the SMEFT is matched on to EFT with only SM quarks but top-quark, the photon and the gluons. Subsequently QCD and QED evolution is performed down to scales $O(1 \mathrm{GeV})$.

Step 3: Around scales $O(1 \mathrm{GeV})$ the matching to the theory of mesons is performed and meson evolution to the factorization scale is performed.

Step 4: The matrix elements of all operators are calculated in the large $N$ limit, that is using factorization of matrix elements into products of currents or densities.

We do not claim that these are all QCD effects responsible for non-leptonic transitions but these evolutions based entirely on non-factorizable QCD effects both at short distance and long distance scales appear to be the main bulk of QCD dynamics responsible for the $\Delta I=1 / 2$ rule, $\varepsilon^{\prime} / \varepsilon$ and $K^{0}-\bar{K}^{0}$ mixing.

\subsection{Past successes of DQCD}

Past successes of this approach have been recently reviewed in [2]. In particular the nonperturbative parameter $\hat{B}_{K}$ has been predicted already in 1987 to be close to its large $N$ value 0.75 and found in 2014 with higher precision to be $\hat{B}_{K}=0.73 \pm 0.02$ [20] in a very good agreement with the present FLAG average $\hat{B}_{K}=0.762 \pm 0.010$ [46].

DQCD also allowed for the first time to identify already in 1986 the dominant mechanism behind the $\Delta I=1 / 2$ rule [18]. In the framework of DQCD the current-current operators and not QCD penguins are responsible dominantly for this rule. In fact this dynamics is rather simple and follows the general pattern outlined above. It is just short distance (quark-gluon) evolution of current-current operators down to scales $O(1 \mathrm{GeV})$ followed by meson evolution down to scales $O\left(m_{\pi}\right)$ at which the hadronic matrix elements factorize and can easily be 
calculated. Improved calculations in 2014 [20] resulted in

$$
\left(\frac{\operatorname{Re} A_{0}}{\operatorname{Re} A_{2}}\right)_{\mathrm{DQCD}}=16.0 \pm 1.5, \quad\left(\frac{\operatorname{Re} A_{0}}{\operatorname{Re} A_{2}}\right)_{\text {lattice } \mathrm{QCD}}=31.0 \pm 11.1,
$$

where we also give the result from the RBC-UKQCD collaboration [6]. Also lattice result is governed by current-current operators. But the uncertainty is still very large and it will be interesting to see whether the lattice QCD will be able to come closer to the data

$$
\left(\frac{\operatorname{Re} A_{0}}{\operatorname{Re} A_{2}}\right)_{\exp }=22.35
$$

than it is possible using DQCD.

I doubt that the remaining piece can be fully explained by NP as this would lead to a large fine-tuning in $\Delta M_{K}$ as demonstrated in [47]. But as analysed in that paper a colour octet of heavy gluons $\left(G^{\prime}\right)$ could bring the theory closer to the data. It is however likely that also final state interactions (FSI) as stressed by Pich and collaborators and additional subleading corrections not included in the DQCD result in (11) could be responsible for the missing piece. Yet, I do not think that the present analytic methods like DQCD or the methods advocated by Pich and collaborators, as reviewed recently in [12], are sufficiently powerful to answer the question at which level NP enters the amplitudes $\operatorname{Re} A_{0}$ and $\operatorname{Re} A_{2}$. Here lattice QCD should provide valuable answers and I am looking forward to improved results on these two amplitudes from RBC-UKQCD collaboration and other lattice groups. This would provide two additional important constraints on NP models.

DQCD is really not QCD, the theory of quarks and gluons, but as we have seen above and we will see below a successful low energy approximation of QCD. Moreover, it has several virtues:

- It is an efficient approximate method for obtaining results for non-leptonic decays, years and even decades before useful results from numerically sophisticated and demanding lattice calculations could be obtained.

- It is the only existing method allowing to study analytically the dominant dynamics below $1 \mathrm{GeV}$ scale, represented by the meson evolution, which turns out to have the pattern of operator mixing, both for SM and BSM operators, to agree with the one found perturbatively at short distance scales. This allows for satisfactory, even if approximate, matching between Wilson coefficients and hadronic matrix elements.

- It provides insight in the purely numerical results obtained by lattice QCD for both $K^{0}-\bar{K}^{0}$ hadronic matrix elements of BSM operators and of SM QCD and electroweak penguin operators relevant for $\varepsilon^{\prime} / \varepsilon$. See below.

- Most importantly it allowed already now to evaluate $K \rightarrow \pi \pi$ matrix elements of all BSM operators providing in this manner for the first time a global view on NP contributions to $\varepsilon^{\prime} / \varepsilon$.

\section{More on $\varepsilon^{\prime} / \varepsilon$ Anomaly}

While the results based on the hadronic matrix elements from RBC-UKQCD lattice collaboration, suggest some evidence for the presence of NP in hadronic $K$ decays, the large uncertainties in the hadronic matrix elements in question do not yet preclude that eventually the SM will agree with data. In this context the upper bounds on the matrix elements of the dominant penguin operators from DQCD [10] are important as they give presently the 
strongest support to the anomaly in question, certainly stronger than present lattice results. To see this in explicit terms let us look at the parameters $B_{6}^{(1 / 2)}$ and $B_{8}^{(3 / 2)}$ that represent the relevant hadronic matrix elements of the QCD penguin operator $Q_{6}$ and the electroweak penguin operator $Q_{8}$, respectively.

In the strict large $N$ limit $[19,44,48]$ one simply has $B_{6}^{(1 / 2)}=B_{8}^{(3 / 2)}=1$, but RBCUKQCD results $[6,7]$ imply $[8,49]$

$$
B_{6}^{(1 / 2)}=0.57 \pm 0.19, \quad B_{8}^{(3 / 2)}=0.76 \pm 0.05, \quad(\mathrm{RBC}-\mathrm{UKQCD}),
$$

and this suppression of both parameters below unity, in particular of $B_{6}^{(1 / 2)}$, is the main origin of the strong suppression of $\varepsilon^{\prime} / \varepsilon$ within the SM below the data. Yet in view of the large error in $B_{6}^{(1 / 2)}$ one could be sceptical about claims made by me and my collaborators that there is $\mathrm{NP}$ in $\varepsilon^{\prime} / \varepsilon$. Future lattice results could in principle raise $B_{6}^{(1 / 2)}$ towards its large $N$ value and above $B_{8}^{(3 / 2)}$ bringing the $\mathrm{SM}$ result for $\varepsilon^{\prime} / \varepsilon$ close to its experimental value.

However, the analyses of $B_{6}^{(1 / 2)}$ and $B_{8}^{(3 / 2)}$ within DQCD in $[10,11]$ show that such a situation is rather unlikely. Indeed, in this approach going beyond the strict large $N$ limit one can understand the suppression of $B_{6}^{(1 / 2)}$ and $B_{8}^{(3 / 2)}$ below the unity as the effect of the meson evolution from scales $\mu=O\left(m_{\pi}\right)$ at which $B_{6}^{(1 / 2)}=B_{8}^{(3 / 2)}=1$ is valid to $\mu=O(1 \mathrm{GeV})$ at which Wilson coefficients of $Q_{6}$ and $Q_{8}$ are evaluated [10]. This evolution has to be matched to the usual perturbative quark evolution for scales higher than $1 \mathrm{GeV}$ and in fact the supressions in question and the property that $B_{6}^{(1 / 2)}$ is more strongly suppressed than $B_{8}^{(3 / 2)}$ are consistent with the perturbative evolution of these parameters above $\mu=O(1 \mathrm{GeV})$. Thus we are rather confident that [10]

$$
B_{6}^{(1 / 2)}<B_{8}^{(3 / 2)}<1 \quad(\text { dual QCD) }
$$

Explicit calculation in this approach gives $B_{8}^{(3 / 2)}\left(m_{c}\right)=0.80 \pm 0.10$. The result for $B_{6}^{(1 / 2)}$ is less precise but in agreement with (13). For further details, see [10].

It should be recalled that in the past values $B_{6}^{(1 / 2)}=B_{8}^{(3 / 2)}=1.0$ have been combined in phenomenological applications with the Wilson coefficients evaluated at scales $\mu=O(1 \mathrm{GeV})$. The discussion above shows that this is incorrect. The meson evolution from $\mu=O\left(m_{\pi}\right)$ to $\mu=O(1 \mathrm{GeV})$ has to be performed and this effect turns out to be stronger than the scale dependence of $B_{6}^{(1 / 2)}$ and $B_{8}^{(3 / 2)}$ in the perturbative regime, where it is very weak.

Additional support for the small value of $\varepsilon^{\prime} / \varepsilon$ in the SM comes from the reconsideration of the role of final state interactions (FSI) in $\varepsilon^{\prime} / \varepsilon$ in [11]. In this paper using the DQCD approach we have demonstrated that in contrast to claims made by chiral perturbation theory practitioners, in particular Pich and collaborators as summarised recently in [12], FSI are not expected to be important for $\varepsilon^{\prime} / \varepsilon$. But we agree with these authors that FSI are likely to be important for the $\Delta I=1 / 2$ rule. I should remark that in private conversations the authors of [12] disagree with our arguments and are convinced that FSI enhance the QCD penguin contribution to $\varepsilon^{\prime} / \varepsilon$ bringing it within the SM to agree with the data. Unfortunately no public response in arxiv to our claim in [11] has been made by these authors and they were unable to demonstrate in private conversations that we are wrong. While lattice QCD calculations are likely to resolve this controversy in due time, it would be desirable if other experts had a look at [11] in order to confirm our claim or invalidate it explicitly in public.

But the controversy on FSI is not the whole story. Also the existence of meson evolution, crucial in the DQCD approach, has been questioned over the last 30 years by some chiral and lattice experts. We have demonstrated in [50] that meson evolution is crucial in reproducing the results for four BSM $K^{0}-\bar{K}^{0}$ matrix elements from lattice QCD (ETM, SWME and 
RBC-UKQCD) collaborations [51-53]. The important virtue of this exercise is the absence of FSI so that the meson evolution can be better exposed than in $K \rightarrow \pi \pi$ decays. In this analysis it is also demonstrated that in contrast to $\hat{B}_{K}$, the strict large $N$ limit in the case of the BSM parameters $B_{2-5}$ is a very bad approximation missing lattice results by factors of $2-3$. Including non-factorizable contributions represented by meson evolution allows to understand the lattice data.

The latter analysis demonstrates the importance of the QCD dynamics at scales below $1 \mathrm{GeV}$ and gives additional support to our claim that meson evolution is the dominant QCD dynamics responsible for the $\Delta I=1 / 2$ rule and also the $\varepsilon^{\prime} / \varepsilon$ anomaly. We are not aware of any analytical approach that could provide such insight in lattice QCD results in question. We challenge the chiral perturbation theory experts to provide an insight into the values of $B_{i}$ parameters from Lattice QCD in their framework, in particular without using lower energy constants obtained from lattice QCD. Motivated by this result we now turn our attention to NP.

\section{Master Formula for $\varepsilon^{\prime} / \varepsilon$ beyond the SM}

Having both the RG evolution and all matrix elements at the low-energy scale $\mu$ for the first time at hand allowed us recently [16] to present a master formula for $\left(\varepsilon^{\prime} / \varepsilon\right)_{\mathrm{BSM}}$ that exhibits its dependence on each Wilson coefficient at the scale $\mu_{\mathrm{ew}}$ and consequently is valid in any theory beyond the SM that is free from non-standard light degrees of freedom below the electroweak scale. We will now present this formula. Technical details which led to this formula can be found in [16] and in particular in [17].

Writing $\varepsilon^{\prime} / \varepsilon$ as a sum of the SM and BSM contributions,

$$
\frac{\varepsilon^{\prime}}{\varepsilon}=\left(\frac{\varepsilon^{\prime}}{\varepsilon}\right)_{\mathrm{SM}}+\left(\frac{\varepsilon^{\prime}}{\varepsilon}\right)_{\mathrm{BSM}},
$$

the master formula of [16] for the BSM part then reads

$$
\left(\frac{\varepsilon^{\prime}}{\varepsilon}\right)_{\mathrm{BSM}}=\sum_{i} P_{i}\left(\mu_{\mathrm{ew}}\right) \operatorname{Im}\left[C_{i}\left(\mu_{\mathrm{ew}}\right)-C_{i}^{\prime}\left(\mu_{\mathrm{ew}}\right)\right] \times(1 \mathrm{TeV})^{2},
$$

where

$$
P_{i}\left(\mu_{\mathrm{ew}}\right)=\sum_{j} \sum_{I=0,2} p_{i j}^{(I)}\left(\mu_{\mathrm{ew}}, \mu\right)\left[\frac{\left\langle O_{j}(\mu)\right\rangle_{I}}{\mathrm{GeV}^{3}}\right],
$$

with the sum over $i$ extending over the Wilson coefficients $C_{i}$ of all operators and their chirality-flipped counterparts, that is $36+36^{\prime}$ linearly independent four-quark operators and $1+1^{\prime}$ chromo-magnetic dipole operators. The $C_{i}^{\prime}$ are the Wilson coefficients of the corresponding chirality-flipped operators obtained by interchanging $P_{L} \leftrightarrow P_{R}$. The relative minus sign accounts for the fact that their $K \rightarrow \pi \pi$ matrix elements differ by a sign. Among the contributing operators are also operators present already in the SM but their Wilson coefficients in (16) include only BSM contributions. In view of space limitations we cannot list all these operators here and the readers are invited to look up our last three papers [15-17].

The dimensionless coefficients $p_{i j}^{(I)}\left(\mu_{\mathrm{ew}}, \mu\right)$ include the QCD and QED RG evolution from $\mu_{\mathrm{ew}}$ to $\mu$ for each Wilson coefficient as well as the relative suppression of the contributions to the $I=0$ amplitude due to $\operatorname{Re} A_{2} / \operatorname{Re} A_{0} \ll 1$ for the matrix elements $\left\langle O_{j}(\mu)\right\rangle_{I}$ of all the operators $O_{j}$ present at the low-energy scale. The index $j$ includes also $i$ so that the effect of self-mixing is included. The $P_{i}\left(\mu_{\mathrm{ew}}\right)$ do not depend on $\mu$ to the considered order, because the 
$\mu$-dependence cancels between matrix elements and the RG evolution operator. Moreover, it should be emphasized that their values are model-independent and depend only on the SM dynamics below the electroweak scale, which includes short distance contributions down to $\mu$ and the long distance contributions represented by the hadronic matrix elements. The BSM dependence enters our master formula in (16) only through the Wilson coefficients $C_{i}\left(\mu_{\mathrm{ew}}\right)$ and $C_{i}^{\prime}\left(\mu_{\mathrm{ew}}\right)$. That is, even if a given $P_{i}$ is non-zero, the fate of its contribution depends on the difference of these two coefficients. In particular, in models with exact left-right symmetry this contribution vanishes as first pointed out in [54].

The numerical values of the $P_{i}\left(\mu_{\mathrm{ew}}\right)$ are collected in the tables presented in $[16,17]$. As seen in (17), the $P_{i}$ depend on the hadronic matrix elements $\left\langle O_{j}(\mu)\right\rangle_{I}$ and the RG evolution factors $p_{i j}^{(I)}\left(\mu_{\mathrm{ew}}, \mu\right)$. The numerical values of the hadronic matrix elements rely on lattice QCD in the case of SM operators and DQCD in the case of BSM operators as summarized above.

Inspecting the results in the tables in [17] the following observations can be made, here given in a simplified form in terms of three islands of hadronic matrix elements: SM-like island governed by SM operators $Q_{7,8}$, chromo-magnetic one governed by chromo-magnetic penguin operator $O_{8 g}$ and the BSM-island conquered in [15]:

- The largest $P_{i}$ values on SM-like island can be traced back to the large values of the matrix elements $\left\langle Q_{7,8}\right\rangle_{2}$, the dominant electroweak penguin operators in the SM, and the enhancement by 22 of the $I=2$ contributions relative to $I=0$ ones.

- The small $P_{i}$ values on the chromo-magnetic island are the consequence of the fact that each one is proportional to $\left\langle O_{8 g}\right\rangle_{0}$, which has recently been found to be much smaller than previously expected $[13,14]$. Moreover, as $\left\langle O_{8 g}\right\rangle_{2}=0$, all contributions in this class are suppressed by the factor 22 relative to contributions from other classes.

- The large $P_{i}$ values on the BSM-island can be traced back to the large hadronic matrix elements of scalar and tensor operators calculated recently in [15].

The question then arises which creatures are living on these islands. Here comes the answer.

\section{Lessons from the SMEFT Analysis of $\varepsilon^{\prime} / \varepsilon$}

In [17] we have presented for the first time a model-independent anatomy of the ratio $\varepsilon^{\prime} / \varepsilon$ in the context of the $\Delta S=1$ EFT with operators invariant under QCD and QED and in the context of the Standard Model Effective Field Theory (SMEFT) with the operators invariant under the full SM gauge group. This was only possible thanks to the very recent calculations of the $K \rightarrow \pi \pi$ matrix elements of BSM operators, namely of the chromo-magnetic dipole operators by lattice QCD [13] and DQCD [14] and in particular through the calculation of matrix elements of all four-quark BSM operators, including scalar and tensor operators, by DQCD [15]. Even if the latter calculations have been performed in the chiral limit, they offer for the first time a look into the world of BSM operators contributing to $\varepsilon^{\prime} / \varepsilon$.

Our main goal was to identify those NP scenarios which are probed by $\varepsilon^{\prime} / \varepsilon$ and which could help to explain the emerging anomaly in $\varepsilon^{\prime} / \varepsilon$ discussed above.

In the last three years a number of analyses, addressing the $\varepsilon^{\prime} / \varepsilon$ anomaly in concrete models, appeared in the literature. They are collected in Table 1 of [2] and also listed in [17]. But all these analyses concentrated on models in which NP entered exclusively through modifications of the Wilson coefficients of SM operators. In particular the Wilson coefficient of the dominant electroweak penguin operator $Q_{8}$. Thus these analyses have been performed on the SM-like island. This is a significant limitation if one wants to have a general view of possible BSM scenarios responsible for the $\varepsilon^{\prime} / \varepsilon$ anomaly. In particular, in the absence of 
even approximate values of hadronic matrix elements of BSM operators, no complete modelindependent analysis was possible until recently.

The recent calculations of BSM $K \rightarrow \pi \pi$ matrix elements, in particular of those of scalar and tensor operators in [15] allowed to perform the analysis also on the BSM-island which combined with the EFT and in particular SMEFT technology in [17] widened significantly our view on BSM contributions to $\varepsilon^{\prime} / \varepsilon$. The analysis in [17] has two main virtues:

- It opens the road to the analyses of $\varepsilon^{\prime} / \varepsilon$ in any theory beyond the SM and allows with the help of the master formula in (16) to search very efficiently for BSM scenarios behind the $\varepsilon^{\prime} / \varepsilon$ anomaly. In particular the values of $P_{i}$ collected in these two papers indicate which routes could be more successful than others both in the context of the low-energy EFT and SMEFT. By implementing our results in the open source code flavio [55], testing specific BSM theories becomes particularly simple.

- Through our SMEFT analysis we were able to identify correlations between $\varepsilon^{\prime} / \varepsilon$ and various observables that depend sensitively on the operators involved. Here $\Delta S=2, \Delta C=2$ and electro-magnetic dipole moments (EDM) play a prominent role but also correlations with $\Delta S=1$ and $\Delta C=1$ provide valuable informations. We ask the interested readers to look at our papers.

Our main messages to take home are as follows:

- Tree-level vector exchanges, like $Z^{\prime}, W^{\prime}$ and $G^{\prime}$ contributions (SM-like island), discussed already by various authors and vector-like quarks [56] can be responsible for the observed anomaly but generally one has to face important constraints from $\Delta S=2$ and $\Delta C=2$ transitions as well as direct searches and often some fine tuning is required. Here the main role is played by the electroweak penguin operator $Q_{8}$ with its Wilson coefficient significantly modified by NP.

- Models with tree-level exchanges of heavy colourless or coloured scalars (BSM-island) are a new avenue, opened with the results for BSM operators from DQCD in [15]. In particular scalar and tensor operators, having chirally enhanced matrix elements and consequently large coefficients $P_{i}$, are candidates for the explanation of the anomaly in question. Moreover, some of these models, in contrast to models with tree-level $Z^{\prime}$ and $G^{\prime}$ exchanges, are free from both $\Delta S=2$ and $\Delta C=2$ constraints. The EDM of the neutron is an important constraint for these models, depending on the couplings, but does not preclude a sizable NP effect in $\varepsilon^{\prime} / \varepsilon$.

- Models with modified $W^{ \pm}$or $Z^{0}$ couplings can induce sizable effects in $\varepsilon^{\prime} / \varepsilon$ without appreciable constraints from semi-leptonic decays such as $K^{+} \rightarrow \pi^{+} v \bar{v}$ or $K_{L} \rightarrow \pi^{0} l^{+} l^{-}$. In the case of a SM singlet $Z^{\prime}$ mixing with the $Z^{0}$, sizable $Z^{0}$-mediated contributions are disfavoured by electroweak precision tests. Yet, as discussed in [2] and in references given there, also such models could play some role in understanding the role of NP in $\varepsilon^{\prime} / \varepsilon$. This is in particular the case of model with vector-like quarks [56].

The future of $\varepsilon^{\prime} / \varepsilon$ in the SM and in the context of searches for NP will depend on how accurately it can be calculated. This requires improved lattice calculations not only of the matrix elements of SM operators but also of the BSM ones, which are known presently only from the DQCD approach in the chiral limit. Moreover, the impact of FSI on the values of $P_{i}$ have to be investigated. As these values are dominated by $I=2$ matrix elements we expect that these effects amount to at most $10-20 \%$ of the present values. It is also hoped that lattice QCD will be able to take into account isospin breaking corrections and that other lattice collaborations will attempt to calculate hadronic matrix elements of all relevant operators. In this context we hope that the new analysis of the RBC-UKQCD collaboration 
with improved matrix elements to be expected this year will shed new light on the hinted anomaly. Such future updates can be easily accounted for by the supplementary details on the master formula in the appendices of [17].

On the short-distance side the NNLO results for QCD penguins should be available soon [33]. The dominant NNLO corrections to electroweak penguins have been calculated almost 20 years ago [30]. As pointed out in [17] all the existing estimates of $\varepsilon^{\prime} / \varepsilon$ at NLO suffer from short-distance renormalization scheme uncertainties in the electroweak penguin contributions and also scale uncertainties in $m_{t}(\mu)$ that are removed only in the NNLO matching at the electroweak scale [30]. In the naive dimensional regularization (NDR) scheme, used in all recent analyses, these corrections enhance the electroweak penguin contribution by roughly $16 \%$, thereby leading to a negative shift of $-1.3 \times 10^{-4}$ decreasing further the value of $\varepsilon^{\prime} / \varepsilon$, similarly to isospin breaking effects. This could appear small in view of other uncertainties. However, on the one hand, potential scale and renormalization scheme uncertainties have been removed in this manner and on the other hand, one day such corrections could turn out to be relevant. Finally, the fact that this correction further decreases $\varepsilon^{\prime} / \varepsilon$ within the SM gives another motivation for the search for new physics responsible for it, and thus for our recent analyses.

As far as BSM operators are concerned, a NLO analysis of their Wilson coefficients is in progress, but its importance is not as high as of hadronic matrix elements due to significant additional parametric uncertainties residing in any NP model. In any case, in the coming years the ratio $\varepsilon^{\prime} / \varepsilon$ is expected to play a significant role in the search for NP. In this respect, the results presented in $[16,17]$ will be helpful in disentangling potential models of new $\mathrm{CP}$ violating sources beyond the SM as well as constraining the magnitude of their effects.

\section{Outlook}

Our outlook is very short. The future of $\varepsilon^{\prime} / \varepsilon$, in particular in correlation with rare decays $K^{+} \rightarrow \pi^{+} v \bar{v}$ and $K_{L} \rightarrow \pi^{0} v \bar{v}$, looks great and the coming years should be very exciting. I am looking forward to QCD@Work 2020 when the landscape of NP will be more transparent than it is now.

\section{Acknowledgements}

It is a pleasure to thank the organizers of this workshop for inviting me to this interesting event and for an impressive hospitality. Particular thanks go to my four great collaborators: Jason Aebischer, Christoph Bobeth, Jean-Marc Gérard and David Straub for fantastic time we spent together in the last six months. This research was supported by the DFG cluster of excellence "Origin and Structure of the Universe".

\section{References}

[1] A.J. Buras, J. Girrbach, Rept. Prog. Phys. 77, 086201 (2014), 1306. 3775

[2] A.J. Buras, Acta Phys. Polon. B49, 1043 (2018), 1805 . 11096

[3] J. Batley et al. (NA48), Phys. Lett. B544, 97 (2002), hep-ex/0208009

[4] A. Alavi-Harati et al. (KTeV), Phys. Rev. D67, 012005 (2003), hep-ex/0208007

[5] E. Abouzaid et al. (KTeV), Phys. Rev. D83, 092001 (2011), 1011.0127

[6] Z. Bai et al. (RBC, UKQCD), Phys. Rev. Lett. 115, 212001 (2015), 1505.07863

[7] T. Blum et al., Phys. Rev. D91, 074502 (2015), 1502.00263 
[8] A.J. Buras, M. Gorbahn, S. Jäger, M. Jamin, JHEP 11, 202 (2015), 1507.06345

[9] T. Kitahara, U. Nierste, P. Tremper (2016), 1607.06727

[10] A.J. Buras, J.M. Gerard, JHEP 12, 008 (2015), 1507.06326

[11] A.J. Buras, J.M. Gerard, Eur. Phys. J. C77, 10 (2017), 1603.05686

[12] H. Gisbert, A. Pich, Rept. Prog. Phys. 81, 076201 (2018), 1712.06147

[13] M. Constantinou, M. Costa, R. Frezzotti, V. Lubicz, G. Martinelli, D. Meloni, H. Panagopoulos, S. Simula (ETM), Phys. Rev. D97, 074501 (2018), 1712.09824

[14] A.J. Buras, J.M. Gérard, JHEP 07, 126 (2018), 1803.08052

[15] J. Aebischer, A.J. Buras, J.M. Gérard (2018), 1807 . 01709

[16] J. Aebischer, C. Bobeth, A.J. Buras, J.M. Gérard, D.M. Straub (2018), 1807.02520

[17] J. Aebischer, C. Bobeth, A.J. Buras, D.M. Straub (2018), 1808.00466

[18] W.A. Bardeen, A.J. Buras, J.M. Gérard, Phys. Lett. B192, 138 (1987)

[19] A.J. Buras, J.M. Gérard, Nucl. Phys. B264, 371 (1986)

[20] A.J. Buras, J.M. Gérard, W.A. Bardeen, Eur. Phys. J. C74, 2871 (2014), 1401.1385

[21] A.J. Buras, M. Jamin, M.E. Lautenbacher, P.H. Weisz, Nucl. Phys. B370, 69 (1992)

[22] A.J. Buras, M. Jamin, M.E. Lautenbacher, P.H. Weisz, Nucl. Phys. B400, 37 (1993), hep-ph/9211304

[23] A.J. Buras, M. Jamin, M.E. Lautenbacher, Nucl. Phys. B400, 75 (1993), hep-ph/9211321

[24] M. Ciuchini, E. Franco, G. Martinelli, L. Reina, Phys. Lett. B301, 263 (1993), hep-ph/9212203

[25] A.J. Buras, M. Jamin, M.E. Lautenbacher, Nucl. Phys. B408, 209 (1993), hep-ph/9303284

[26] M. Ciuchini, E. Franco, G. Martinelli, L. Reina, Nucl. Phys. B415, 403 (1994), hep-ph/9304257

[27] M. Ciuchini, E. Franco, V. Lubicz, G. Martinelli, I. Scimemi et al., Nucl. Phys. B523, 501 (1998), hep-ph/9711402

[28] A.J. Buras, M. Misiak, J. Urban, Nucl. Phys. B586, 397 (2000), hep-ph/0005183

[29] C. Bobeth, M. Misiak, J. Urban, Nucl. Phys. B574, 291 (2000), hep-ph/9910220

[30] A.J. Buras, P. Gambino, U.A. Haisch, Nucl. Phys. B570, 117 (2000), hep-ph/9911250

[31] M. Gorbahn, U. Haisch, Nucl. Phys. B713, 291 (2005), hep-ph/0411071

[32] J. Brod, M. Gorbahn, Phys. Rev. D82, 094026 (2010), 1007. 0684

[33] M. Cérdà-Sevilla, M. Gorbahn, S. Jäger, A. Kokulu, J. Phys. Conf. Ser. 800, 012008 (2017), 1611.08276

[34] J. Aebischer, A. Crivellin, M. Fael, C. Greub, JHEP 05, 037 (2016), 1512 . 02830

[35] E.E. Jenkins, A.V. Manohar, P. Stoffer, JHEP 03, 016 (2018), 1709.04486

[36] E.E. Jenkins, A.V. Manohar, M. Trott, JHEP 10, 087 (2013), 1308. 2627

[37] E.E. Jenkins, A.V. Manohar, M. Trott, JHEP 01, 035 (2014), 1310. 4838

[38] R. Alonso, E.E. Jenkins, A.V. Manohar, M. Trott, JHEP 04, 159 (2014), 1312 . 2014

[39] G. 't Hooft, Nucl. Phys. B72, 461 (1974)

[40] G. 't Hooft, Nucl. Phys. B75, 461 (1974)

[41] E. Witten, Nucl. Phys. B160, 57 (1979)

[42] S.B. Treiman, E. Witten, R. Jackiw, B. Zumino, Current Algebra and Anomalies (Singapore, Singapore: World Scientific ( 1985) 537p, 1986)

[43] R.S. Chivukula, J. Flynn, H. Georgi, Phys.Lett. B171, 453 (1986)

[44] W.A. Bardeen, A.J. Buras, J.M. Gérard, Phys. Lett. B180, 133 (1986) 
[45] W.A. Bardeen, A.J. Buras, J.M. Gérard, Nucl. Phys. B293, 787 (1987)

[46] S. Aoki et al., Eur. Phys. J. C77, 112 (2017), 1607.00299

[47] A.J. Buras, F. De Fazio, J. Girrbach, Eur. Phys. J. C74, 2950 (2014), 1404 . 3824

[48] A.J. Buras, J.M. Gérard, Phys. Lett. B192, 156 (1987)

[49] A.J. Buras, D. Buttazzo, J. Girrbach-Noe, R. Knegjens, JHEP 11, 033 (2015), 1503.02693

[50] A.J. Buras, J.M. Gerard (2018), 1804.02401

[51] N. Carrasco, P. Dimopoulos, R. Frezzotti, V. Lubicz, G.C. Rossi, S. Simula, C. Tarantino (ETM), Phys. Rev. D92, 034516 (2015), 1505.06639

[52] B.J. Choi et al. (SWME), Phys. Rev. D93, 014511 (2016), 1509.00592

[53] P. Boyle, N. Garron, J. Kettle, A. Khamseh, J.T. Tsang, EPJ Web Conf. 175, 13010 (2018), 1710.09176

[54] G.C. Branco, J.M. Frere, J.M. Gerard, Nucl. Phys. B221, 317 (1983)

[55] D.M. Straub et al., flavio - flavour phenomenology in the standard model and beyond, https://flav-io.github.io

[56] C. Bobeth, A.J. Buras, A. Celis, M. Jung, JHEP 04, 079 (2017), 1609. 04783 